\title{
An integrated approach to characterization of microbial exudates and investigation of their role in the spatial distribution and transformations of uranium at the mineral-microbe interface
}

Argonne National Laboratory: K. M. Kemner*, E. J. O’Loughlin and S. D. Kelly (Argonne National Laboratory, Biosciences Division, 9700 South Cass Avenue, Argonne, Illinois, 60439, Kemner@anl.gov (630)252-1163

University of Southern California: K. H. Nealson

Research Objective: The long-term aim of this project was to understand the role of microbiota and their polymers (EPS) in controlling the distribution and fates of contaminants in subsurface environments. Additionally, this project also focused on the identification and characterization of extracellular proteins under a variety of growth conditions. Finally, this project sought to develop and advance the use of a variety of synchrotron-based hard-x-ray techniques to address a number of different ERSP elements.

The technical goals related to (1) imaging the spatial distributions of radionuclide and metal contaminants in mineral-microbe systems, and (2) determining the chemical speciation of the contaminants in these systems. The scientific goals related to the determination of the role of microbial exudates in microbial reduction and partitioning of metals and radionuclides between the soluble and solid phases. The specific objectives of the project were to accomplish the following:

1. Develop x-ray and coupled $\mathrm{x}$-ray-electron imaging methods; advance $\mathrm{x}$-ray spectroscopic methods for determining the spatial distribution and chemical speciation of $\mathrm{Fe}$ and $\mathrm{U}$ in mineral-microbe systems; determine the detection limits and spatial resolution of the techniques.

2. Identify the exuded microbial polymers (e.g., polysaccharides, DNA, RNA, and proteins).

3. Determine the types and numbers of aqueous proton binding sites in microbial polymers and relate them to metal binding properties.

4. Determine the spatial distribution and chemical speciation of $U$ exposed to a variety of microbial polymers from $S$. oneidensis MR-1 (and mutants) under conditions that should lead to oxidation or reduction of each metal.

5. Determine the role of microbial polymers as they either promote or hinder $U$ reduction, even after the metal may have been released from (hydr)oxides by microbial reduction.

Research Progress and Implications: This final report summarizes work during FY2005 and 2006.

Mission relevance. This project was designed to generate information about the relationships among microbial metabolic processes, microbial cell microenvironments, and aqueous and solidphase geochemistry related to the sequestration, release, precipitation, solubility, organic complexation, and chemical modification (oxidation-reduction) of metals and radionuclides. Such information is of the utmost importance for a better understanding of biotransformation processes and biogeochemical interactions and for the development of science-based solutions for cleanup of contaminated DOE sites, an effort consistent with the primary focus of the ERSP.

Potentiometric acid-base titration: We began acid-base titrations of EPS from S. oneidensis MR-1 and from P. fluorescens. The aim was to observe and analyze differences in the protonbinding behavior of a background electrolyte, the growth medium for the bacteria, the filtrate 
passing through a $0.2-\mu \mathrm{m}$ filter after onset of stationary phase growth (EPS), and the filtrate passing through a $0.2-\mu \mathrm{m}$ filter after the addition of $\mathrm{Ca}^{2+}$ to EPS (EPS-Ca). In the case of $S$. oneidensis MR-1, the effect of adding suspended ferrihydrite to the system was also investigated, as was the proton sorption capacity of ferrihydrite as a function of ionic strength.

In all samples having bacterial products, the titration seemed not to be completely reversible over the entire $\mathrm{pH}$ range examined $(\mathrm{pH} 2-12)$, suggesting conformational changes of the EPS at $\mathrm{pH}$ 2.0-3.0. In both $S$. oneidensis MR-1 and P. fluorescens growth medium, an abundant low-pH site was significantly decreased in number after bacterial growth. Comparisons of $S$. oneidensis MR-1 minimal salts growth medium, with and without lactate, showed that the low-pH site was due to lactate. A difference in titrations for EPS versus EPS-Ca samples was observed only when ferrihydrite was added, not in the absence of ferrihydrite. Fits of the data show that the concentration of binding sites is close to the sum of the concentrations for EPS-Ca and ferrihydrite samples. This result suggests that the proton-binding effect of ferrihydrite is additive, possibly due to limited interaction between EPS and ferrihydrite or binding of EPS to ferrihydrite through sites other than proton-binding sites, in a way that does not block access to protonbinding sites. For the $P$. fluorescens solutions, an overall increase in proton-binding capacity observed after growth of the bacteria suggests the production of proton-binding material, as the increase in total sites occurs despite a loss of sites in the low-pH region, consistent with the depletion of lactate.

A tentative assignment of the proton-binding sites can be made through comparison of the acid dissociation constants to those of aqueous acids. We tentatively assign three types of binding sites within the EPS at 3.4-3.8, 6.0-6.8, and 9.1-9.4 as carboxyl, phosphoryl, and hydroxyl sites, respectively. These acid-base titrations, followed by U LIII edge x-ray absorption fine structure (XAFS) measurements directly probed steric chemical interactions between U and the microbial exudates, and provided insight to the role of EPS in the chemical speciation of U.

$\underline{X-r a y ~ a b s o r p t i o n ~ f i n e ~ s t r u c t u r e ~(m i c r o) s p e c t r o s c o p y ~ a n a l y s i s ~ o f ~ u r a n i u m, ~ a n d ~ b a c t e r i a l ~ c e l l s ~ a n d ~}$ their associated EPS adhered to iron (hydr)oxides: We performed $\mathrm{U}_{\mathrm{III}}$ edge XAFS investigations at the MRCAT insertion device beam line at the Advanced Photon Source (APS) on bulk samples containing either lepidocrocite or ferrihydrite powders and $U(0.05 \mathrm{mM})$ in solutions of either minimal salts bacterial growth media or EPS (i.e., the filtrate passing through a 0.2 micron filter after the onset of stationary phase growth) to directly probe the interactions between U, EPS from aerobically grown $S$. oneidensis MR-1, and iron (hydr)oxides (see figure). Measurements were made in the fluorescence mode and utilized a harmonic rejection mirror. Results from $\mathrm{U} \mathrm{L}_{\mathrm{III}}$-edge XAFS measurements of EPS identify both $\mathrm{C}$ and $\mathrm{P}$ backscattering atoms around $U$, consistent with the presence of carboxyl and phosphoryl functional groups in the EPS. XAFS measurements of the bulk lepidocrocite in the presence of aerobically grown EPS or growth media indicate less interaction in the solid phase between $U$ and lepidocrocite than between $U$ and phosphates. Further analyses of these data are consistent with some precipitation of hydrous uranyl phosphate. In contrast, XAFS measurements of bulk ferrihydrite in the presence of aerobically produced EPS indicate more U-Fe correlations in the higher coordination shells around $U$ than with phosphoryl functional groups associated with the EPS. These XAFS results indicate that the ability of EPS to complex uranium is dependent on the iron (hydr)oxide mineral surfaces that are present in the system.

The combination of the high brilliance of the APS synchrotron and the use of high-resolution zone plates for focusing improves $\mathrm{x}$-ray microimaging experiments of mineral-microbe-metal systems substantially. We have used SEM, XRF microscopy (at the XOR 2ID-D microbe beam line), and bulk XAFS (at the MRCAT 10ID beam line) to investigate the interactions between U, 
S. oneidensis MR-1, and amorphous lepidocrocite thin films under anoxic conditions. Results from these studies demonstrate the ability of XRF microscopy to identify the location of U, relative to cells adhered to Fe (hydr)oxide thin films, and the ability of scanning electron microscopy (SEM) to image EPS in close association with cells adhered to the films. XRF microscopy results for samples incubated for one day indicate that $U$ is associated with the bacteria and adjacent extracellular material. XRF microscopy results for samples incubated for three days indicate that $\mathrm{U}$ is dispersed throughout the biofilm and coincident with the location of EPS and biomineralization products. Results from $\mathrm{U}_{\mathrm{III}}$-edge XANES measurements of these films indicate that the $U$ is not reduced after three days of incubation. Possible explanations for the lack of discernable reduction of $U$ include (1) a lag in anaerobic respiration of the $S$. oneidensis MR-1, (2) reoxidation of the $\mathrm{U}$ by the ferric (hydr)oxide film, and (3) binding of the U(VI) by EPS with chemical and physical hindering of the bioreduction process.

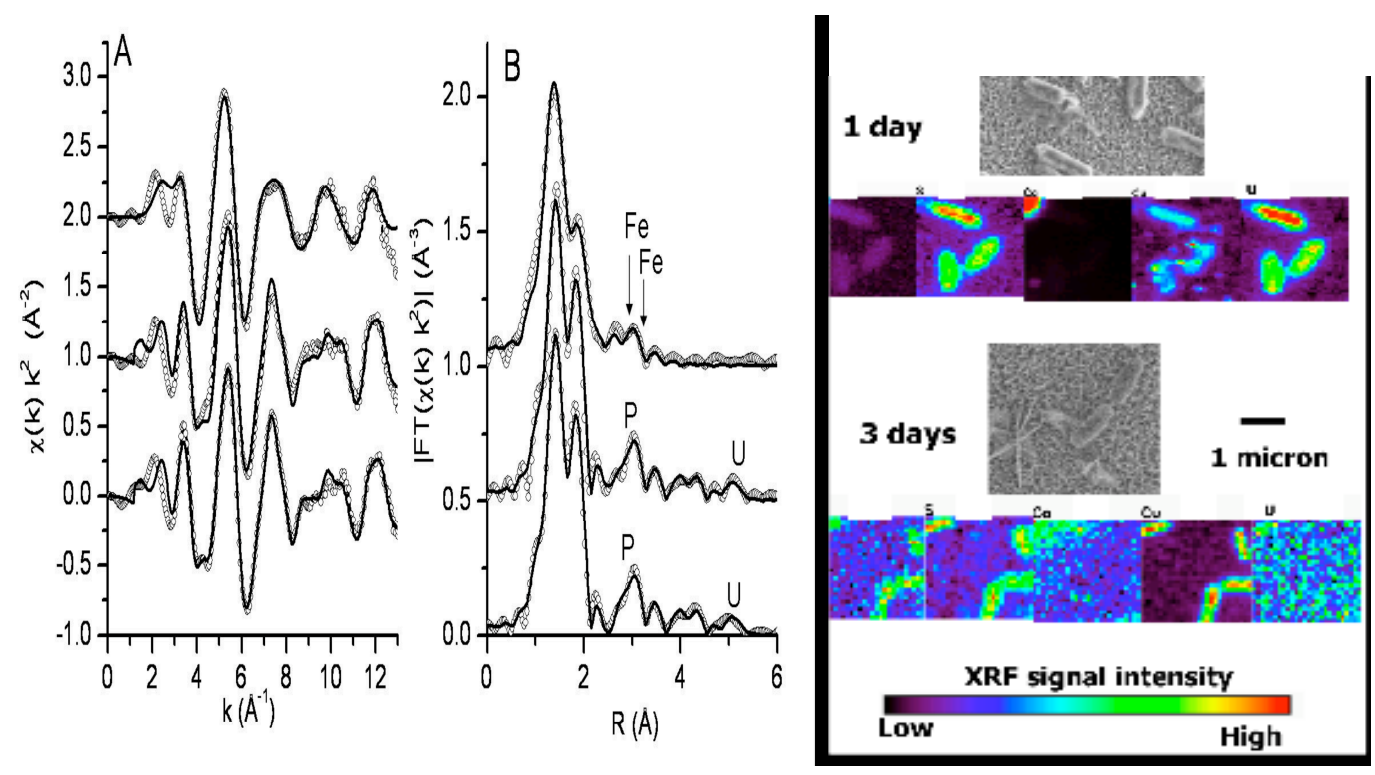

EXAFS and Fourier transform of U LIII-edge EXAFS data (left) measured on $U+$ ferrihydrite $+E P S$ (top), U+Lepidocrocite $+E P S$ (middle), and U+EPS (bottom). Scanning electron microscopy and XRF microscopy results (right) of 1-day and 3-day incubations of $S$. oneidensis MR-1 deposited on lepidocrocite thin films deposited on kapton.

c-type cytochrome-dependent formation of U(IV) nanoparticles by Shewanella oneidensis: Working in collaboration with colleagues at the Pacific Northwest National Laboratory, we showed that $c$-type cytochromes of a dissimilatory metal reducing bacterium, Shewanella oneidensis MR-1 are essential for the reduction of $\mathrm{U}(\mathrm{VI})$ and formation of extracelluar $\mathrm{UO}_{2}$ nanoparticles. In particular, two outer membrane (OM) decaheme cytochromes $\mathrm{MtrC}$ and OmcA, previously implicated in $\mathrm{Mn}(\mathrm{IV})$ and $\mathrm{Fe}(\mathrm{III})$ reduction, significantly affected the U(VI) reduction rates and also displayed differences in subcellular localization of $\mathrm{UO}_{2}$. Similar to the wild type, the mutants accumulated $\mathrm{UO}_{2}$ nanoparticles extracellularly to high densities in association with an exopolymeric substance (EPS). In wild type cells, this $\mathrm{UO}_{2}$-EPS matrix exhibited glycocalyx-like properties, and contained multiple elements of the OM, polysaccharide, and heme containing proteins. Using a novel combination of methods including synchrotron-based X-ray fluorescence microscopy and high-resolution immune-electron 
microscopy, we demonstrated a close association of the extracellular $\mathrm{UO}_{2}$ nanoparticles with $\mathrm{MtrC}$ and OmcA. This was the first study to directly localize the OM-associated cytochromes with extracellular EPS, as a glycocalyx-like structure, which contains biogenic $\mathrm{UO}_{2}$ nanoparticles.

\section{Planned Activities:}

Although funding for this project has been terminated, we intend to submit a follow up proposal to further investigate these observations. Specifically, we have observed similar lags in reduction of $\mathrm{Fe}$ in fine-particle lepidocrocite. In addition, past investigations showed that poorly crystalline ferrihydrite strongly inhibited bioreduction and reoxidized U(IV). To address this issue, we will propose to investigate longer incubation times, the use of different bacterial strains (S. putrefaciens CN32, Geobacter spp., and Anaeromyxobacter spp.), and alternative iron (hydr)oxide thin films for these experiments.

\section{Information Access:}

Publications:

K. M. Kemner, E. J. O’Loughlin, S. D. Kelly, B. Ravel, M. Boyanov, D. Sholto-Douglas, B. Lai, R. Cook, E. Carpenter, V. Harris, K. H. Nealson, "XAFS And X-ray And Electron Microscopy Investigations Of Radionuclide Transformations At The Mineral-Microbe Interface" submitted to American Institute of Physics International XAFS Conference Proceedings.

M. J. Marshall, A. S. Beliaev, A. C. Dohnalkova, D. W. Kennedy, L. Shi, Z. Wang, M. I. Boyanov, B. Lai, K. M. Kemner, J. S. McLean, S. B. Reed, D. E. Culley, V. L. Bailey, C. J. Simonson, D. A. Saffarini, M. F. Romine, Y. Gorby, J. M. Zachara, J. K. Fredrickson, "c-Type Cytochrome-Dependent Formation of U(IV) Nanoparticles by Shewanella oneidensis," PloS Biology, Vol. 4, Issue 8.

K. M. Kemner, S. D. Kelly, B. Lai, J. Maser, E. J. O’Loughlin, D. Sholto-Douglas, Z. Cai, M. A. Schneegurt, C. F. Kulpa, Jr., K. H. Nealson, "Elemental and Redox Analysis of Single Bacterial Cells by X-ray Microbeam Analysis," SCIENCE, 306 686-687, 2004

K. M. Kemner, S. D. Kelly, E. J. O’Loughlin, T. Khare, Y. Londer, M. Schiffer, L. A. Moe, B. G. Fox, M. I. Donnelly, and C. S. Giometti, "XRF and XAFS Analysis of Electrophoretically Isolated Nondenatured Proteins," Physica Scripta T115 940-942, 2005.

K. M. Kemner, E. J. O’Loughlin, S. D. Kelly, M. I. Boyanov, "Synchrotron x-ray investigations of mineral-microbe-metal interactions," Elements August, 2005, pg. 217-221.

K. M. Kemner and S. D. Kelly, "Synchrotron-based Techniques for Monitoring Metal Tranformations," Manual of Environmental Microbiology, Third Edition.

Presentations:

K. M. Kemner, "Molecular Environmental Science: From X-rays to Biogeochemistry to Beethoven," Fermi National Laboratory Colloquium, Batavia, Illinois, September 20, 2006. (invited)

K. M. Kemner, "Biogeochemistry studies using X-ray fluorescence micro(spectro)scopy," Hasy Lab, Hamburg, Germany, September 9-13, 2006. (invited)

K. M. Kemner, "X-ray microscopy analysis of bacterial cells," Microscopy and Microanalysis 2006, Chicago, Illinois, August 3, 2006. (invited)

K. M. Kemner, "The role of synchrotron-based research on soil physicochemical and biological interfacial interactions pertaining to metals and metalloids in the environment," International Union of Soil Science World Congress Meeting, Philadelphia, Pennsylvania, July 10-14, 2006. (invited) 
K. M. Kemner, "From X-rays to Biogeochemistry to Beethoven," Advanced Photon Source Annual Users' Meeting, Argonne National Laboratory, Chicago, Illinois, May 3, 2006. (invited)

K. M. Kemner, "Environmental Contamination: Elucidating the Role of Bacteria," NIST Colloquium, Gaithersburg, MD, March 17, 2006. (invited)

K. M. Kemner, "Using synchrotron light to investigate the spatial distributions and transformations of elements at the bacteria-geosurface interface," International Pacifichem Conference, Honolulu, Hawaii, December 16, 2005. (invited)

K. M. Kemner, "X-ray imaging and spectroscopy for making insitu measurements of biological and physicochemical properties and processes," National Academy of Sciences Soil Workshop, National Academy of Sciences, Washington, D.C., December 14, 2005. (invited)

K. M. Kemner, M. Boyanov, E. J. O’Loughlin, S. D. Kelly, B. Ravel, "The use of synchrotronbased techniques for biogeoscience research," Synchrotron Environmental Science-III Conference, Brookhaven National Laboratory, Upton, NY, September 19-21, 2005. (invited)

K. M. Kemner, "The application of x-ray microbeams to environmental and life sciences," X-ray Physics Gordon Research Conference, Colby-Sawyer College, New London, New Hampshire, August 7-12, 2005. (invited)

K. M. Kemner, "X-ray microprobe investigations of mineral-metal-microbe interfaces," International Goldschmidt Conference, Moscow, Idaho, May 21-26, 2005. (invited)

K. M. Kemner, "Synchrotron-based studies of Geomicrobiology," APS/Users Monthly Operations Meeting, Argonne National Laboratory, Argonne, Illinois, December 16, 2004. (invited)

K. M. Kemner, E. J. O’Loughlin, S. D. Kelly, B. Ravel, M. Boyanov, D. Sholto-Douglas, B. Lai, R. Cook, E. Carpenter, V. Harris, K. H. Nealson, "XAFS And X-ray And Electron Microscopy Investigations Of Radionuclide Transformations At The Mineral-Microbe Interface" $13^{\text {th }}$ International XAFS Conference, Stanford University, Palo Alto, CA, July 914, 2006.

D. W. Kennedy, M. J. Marshall, A. S. Beliaev, A. C. Dohnalkova, L. Shi, Z. Want, M. I. Boyanov, B. Lai, K. M. Kemner, J. S. McLean, S. B. Reed, V. L. Bailey, D. A. Saffarini, M. F. Romine, J. M. Zachara, and J. K. Fredrickson, "The Role of Shewanella oneidensis MR-1 Outer Membrane $c$-Type Cytochromes in Extracellular U(IV)O2 Nanoparticle Formation," American Society of Microbiology Meeting, May 21-25, 2006, Orlando, FL.

M. J. Marshall, A. S. Beliaev, D. W. Kennedy, A. E. Plymale, A. C. Dohnalkova, L. Shi, Z. Wang, M. I. Boyanov, B. Lai, K. M. Kemner, J. S. McLean, S. B. Reed, D. E. Culley, B. L. Bailey, C. J. Simonson, D. A. Saffarini, M. F. Romine, Y. A. Gorby, J. M. Zachara, and J. K. Fredrickson, "Biomolecular Mechanisms of $\mathrm{U}_{(I V)} \mathrm{O}_{2}$ and $\mathrm{Tc}(\mathrm{IV}) \mathrm{O}_{2}$ Nanoparticle Formation by Shewanella oneidensis MR-1," Environmental Remediation Science Program PI Meeting, April 3-5, 2006, Arlie, VA.

M. J. Marshall, D. W. Kennedy, A. C. Dohnalkova, A. E. Plymale, D. A. Saffarini, M. I. Boyanov, K. M. Kemner, B. Lai, S. B. Reed, D. E. Culley, M. F. Romine, A. S. Beliaev, J. M. Zachara, and J. K. Fredrickson, "Characterization of the c-type Cytochromes and the Type II Secretion System of Shewanella oneidensis MR-1 in Radionuclide Reduction and Localization,” ISSM/ISEB Conference, Jackson Hole, Wy. 